\title{
Effectiveness factor of two-dimensional ring-shaped catalyst pellets
}

\author{
Mohammad Asif*, Ahmed A. Ibrahim, Yahya A. Mohammed, Ebrahim H. Al-Ghurabi \\ King Saud University, Department of Chemical Engineering, PO Box 800, Riyadh 11421, KSA \\ "Corresponding author: e-mail: masif@ksu.edu.sa
}

\begin{abstract}
The use of hollow catalytic support improves the utilization of the catalytic material because of the absence of the pellet core, and moreover ensures low reactor pressure drop owing to enhanced bed voidage. In this study, the expressions for the efficient computation of the effectiveness factor are derived for a ring-shaped catalyst pellet undergoing first-order irreversible reaction. The methodology consists of using solutions of one-dimensional problems to remove non-homogeneous boundary conditions. The expressions obtained exhibit significantly faster convergence behavior than those reported in literature. The shape parameters, namely, the height-to-diameter ratio and the inner-to-outer radii ratio, significantly affect the catalyst utilization, such that several-fold improvement in the effectiveness factor is achievable.
\end{abstract}

Keywords: hollow catalyst pellet, effectiveness factor, Thiele modulus, convergence behavior, analytical solution.

\section{INTRODUCTION}

The effective use of a catalytic material is an important challenge for the successful design and scale-up of any heterogeneous reactor system. A portion of the catalytic material, mainly toward the core of the pellet, usually remains unused owing to the intra-particle diffusional resistance. The simplest solution to this challenge is to use a small catalytic support to ensure a large specific surface area; this solution can help lower the effect of the internal diffusional resistance, thus leading to a high effectiveness factor and an improved utilization of the catalytic material. The approach is simple but leads to high pressure drops when implemented on an actual large-scale industrial heterogeneous reactor, thereby ultimately translating into unacceptably high energy costs. Therefore, any strategy for the effective utilization of the catalytic material must also simultaneously address the issue of the high pressure drop in heterogeneous catalytic reactors.

The enhancement in the effectiveness factor has attracted significant attention in literature. For example, a non-uniform distribution of the catalytic material is one of the strategies proposed in the literature ${ }^{1-6}$. However, the successful implementation of such a strategy in an actual reactor is challenging. Another approach suggested involves a careful design of the structure of the catalyst matrix by mixing materials of two different diffusivities. The low diffusivity material with small pore sizes affords large surface areas, but their utility is limited because of the high internal mass transport resistances. An optimized addition of materials of high diffusivity with controlled pore size and porosity can nonetheless substantially improve the utilization of the porous catalytic material ${ }^{7-10}$. Both strategies mentioned in the foregoing require careful design and repeated trials prior to actual implementation. Another recent study clearly showed that the conversion level in a fixed bed reactor can be significantly enhanced by simply using a hollow non-spherical catalytic support ${ }^{11}$. The enhancement in the effectiveness factor for the case of a hollow support is obvious, as the removal of the interior of the catalyst pellet, which cannot be accessed by the reactant in a diffusion-controlled reaction, ensures an improved utilization of the catalytic material while generating an additional surface that further augments the transport of the reactant inside the catalyst.

In addition to high conversion, the pressure drop, which is of paramount importance to the operation of any industrial heterogeneous fixed-bed reactor, is also substantially lowered because of the enhanced bed void fraction of the fixed bed containing hollow packing. Any increase in the bed void fraction, however small, leads to a substantial pressure-drop reduction in the fixed-bed reactors owing to a strong dependence of the pressuredrop on the bed void fraction. Therefore, the lowering of the pressure drop along the reactor length also helps improve the conversion level in gas-phase reactions ${ }^{12}$. Moreover, strategies for enhancing the effectiveness factor suggested that the spherical support can be easily extended to a hollow non-spherical support.

With the advantages inherent to hollow, non-spherical shapes, several examples of their applications are found in industrial practice, such as LP Series rings from DuPont (MECS $\AA$ catalysts) in the production of sulfuric acid and ammonia decomposition catalyst from Pingxiang Hual$\operatorname{ian}^{13,14}$. Several ring-shaped catalysts are available from Haldor Topsoe ${ }^{15}$. Examples of these catalysts are TK-250 for hydrogenenation, HTG-1 used in chlorine absorption for feed purification, SSK-10 sour shift catalyst, CK-322 and CK-428 for VOC removal, PK-5 and PK-7R for conversion of carbon oxides to methane, TK-222 for treating tail gases derived from Claus or similar units, TK-335 and TK-337 for diesel and renewable fuel hydro-treating, and RKS-2 and RKA-02 for secondary and auto-thermal reforming. Similarly, secondary reforming catalyst series, namely, NIAP-20 $(19.5 \mathrm{~mm} \times 14 \mathrm{~mm} \times 8.5 \mathrm{~mm})$ and NIAP-20-01 $(14.5 \mathrm{~mm} \times 12 \mathrm{~mm} \times 6.5 \mathrm{~mm})$, and primary reforming catalyst, NIAP-18 $(14.5 \mathrm{~mm} \times 12 \mathrm{~mm} \times 6.5$ $\mathrm{mm}$ ), are available from Matros Technologies ${ }^{16}$. Several other examples of the use of ring-shaped catalyst pellets are found in actual industrial practice.

Despite the numerous applications of non-spherical hollow catalysts in process industries, attempts to rigorously model their behavior are scarce. Only recently, Asif ${ }^{11}$ presented a heterogeneous model using hollow cylindrical catalyst for ethylene oxidation. A significant reduction in the catalyst requirement as well as the pressure drop was noted. Moreover, a retrofitting strategy 
based on the use of hollow non-spherical biocatalyst for glucose isomerization was recently conducted ${ }^{\mathbf{1 7}}$. The shape parameters of the biocatalyst were found to significantly influence the performance level of the reactor.

The heterogeneous reactor modeling containing even a simple hollow support matrix is an easy task because it requires the solution of a two-dimensional partial differential equation that governs the concentration distribution in the catalyst matrix. This solution should then be coupled with the fluid-phase reactant concentration distribution to predict the behavior of the reactor.

In the present study, an efficient analytical expression is derived to evaluate the potential of ring-shaped catalyst pellets in enhancing the conversion in heterogeneous reactors. This enhancement will obviate the need to develop codes for the solution of two-dimensional partial differential equations, which is often extremely time consuming and requires high-level mathematical skill. The case of linear kinetics is considered in the following parts, and efficient analytical expressions for the effectiveness factor are developed. The enhancement in the effectiveness factor caused by the use of the ring-shaped catalyst pellet is systematically investigated in terms of its relevant shape parameters. The results of such a study can be immediately used to retrofit and optimize the shape of the support geometry and thus enhance the conversion level in the heterogeneous reactor.

\section{MATHEMATICAL MODEL AND SOLUTION APPROACHES}

A simple schematic of a two dimensional ring-shaped catalyst pellet is shown in Figure 1. The steady state concentration profile of a reactant in such a geometry with a first-order irreversible chemical reaction is given by $\left[\frac{1}{\rho} \frac{\partial}{\partial \rho}\left(\rho \frac{\partial v}{\partial \rho}\right)\right]+\frac{1}{\gamma^{2}}\left[\frac{\partial^{2} v}{\partial \xi^{2}}\right]-\phi^{2} v=0$

At $\rho=\kappa ; \quad v=1$ for all $\xi$

At $\rho=1 ; v=1$ for all $\xi$

At $\xi=0 ; \quad \frac{\partial v}{\partial \xi}=0$ for all $\varrho$

At $\xi=1 ; \quad v=1$ for all $\varrho$

where

$v=\frac{C}{C_{o}} ; \quad \rho=\frac{r}{R_{o}} ; \quad \xi=\frac{z}{(H / 2)} ; \quad \gamma^{2}=\left(\frac{D_{r}}{D_{z}} \frac{(H / 2)^{2}}{R_{o}^{2}}\right) ; \quad \kappa=\frac{R_{i}}{R_{o}} ; \quad \phi^{2}=\left(\frac{k_{1} R_{0}^{2}}{D_{r}}\right)$

where $v, \varrho$, and $\xi$ are dimensionless variables representing concentration distribution, radial, and axial distances,

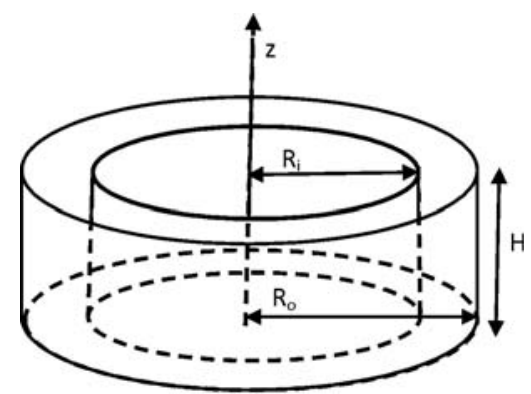

Figure 1. A simple geometrical representation of a twodimensional ring-shaped catalyst pellets finite hollow cylinder respectively. $x$ and $\gamma$ are characteristic shape parameters of the catalyst pellet; $\phi$ is the Thiele modulus; $C_{o}$ is the bulk concentration of the reactant at the surface of the catalyst pellet; $H$ is the height; $R_{i}$ and $R_{o}$ are the inner and outer radii, respectively; $D_{r}$ and $D_{z}$ are the effective diffusivities of the reactant in the radial and longitudinal directions, respectively; $k_{1}$ is the first-order rate constant.

Analytical solutions of the abovementioned twodimensional partial differential equation and evaluation of effectiveness factors have been investigated. Most analytical expressions involve the summation or product of one or more infinite series. For some parameter values, the series can exhibit a convergence problem. Such series convergence issues related to the evaluation of the effectiveness factor can add further constraints to the already-complex heterogeneous reactor modeling. While remedial solutions to convergence problems can be sought in different ways, the methodology used to obtain the solution itself nonetheless primarily affects their convergence behavior. Asif ${ }^{\mathbf{1 8}}$ showed that expressions obtained using a particular solution approach yields a substantially faster convergence behavior than all other analytical expressions proposed in literature. The same methodology is extended to the case of the finite hollow cylindrical catalyst pellet in this study. The method consists of using the particular solution to remove the non-homogeneity associated with boundary conditions. The analytical expressions obtained are then compared with other expressions reported in literature.

On the one hand, the non-homogeneous boundary conditions can be removed by the particular solution approach. If the particular solution based on the solution of an infinite hollow cylinder is used, then the non-homogenous boundary conditions (2a) and 2(b) can be removed. On the other hand, the non-homogenous boundary conditions represented by $(2 \mathrm{c})$ and $(2 \mathrm{~d})$ can be removed through the particular solution based on the solution of an infinite disk. Both approaches, termed as Approach 1 and Approach 2, are considered in the following sections.

\section{Approach 1}

The governing differential equation for a one-dimensional infinite hollow cylinder is given by

$\frac{1}{\rho} \frac{\partial}{\partial \rho}\left(\rho \frac{\partial v_{1}}{\partial \rho}\right)-\phi^{2} v_{1}=0$

At $\rho=\kappa ; \quad v_{1}=1$ for all $\xi$

At $\rho=1 ; \quad v_{1}=1$ for all $\xi$

The solution of Eqs. (4a)-(4c) can be given by

$v_{l}(\rho)=\frac{P_{0}(\phi, \phi \rho)-P_{0}(\kappa \phi, \phi \rho)}{P_{0}(\phi, \kappa \phi)}$

where

$P_{0}(a, b)=I_{0}(a) K_{0}(b)-I_{0}(b) K_{0}(a)$

while $I_{j}$ and $K_{j}$ are $j^{\text {th }}$ order modified Bessel function of the first and second kinds, respectively. Introducing the following substitution

$v(\rho, \xi)=\Psi_{1}(\rho, \xi)+v_{1}(\rho)(6)$ to Eqs. (1) and (2) yields 
$\left[\frac{\partial^{2} \Psi_{1}}{\partial \rho^{2}}+\frac{1}{\rho} \frac{\partial \Psi_{1}}{\partial \rho}\right]+\frac{1}{\gamma^{2}}\left[\frac{\partial^{2} \Psi_{1}}{\partial \xi^{2}}\right]-\phi^{2} \Psi_{1}=0$

At $\rho=\kappa ; \quad \Psi_{1}=0$

At $\rho=1 ; \quad \Psi_{1}=0$

At $\xi=0 ; \frac{d \Psi_{1}}{d \xi}=0$

At $\xi=1 ; \quad \Psi_{1}=1-v_{1}$

Using the method of the separation of variables ${ }^{\mathbf{1 9}}$, the solution of Eqs. (7) and (8) can be given as

$\psi_{1}(\rho, \xi)=\sum_{n=1}^{\infty}\left[\frac{\pi^{2}}{2} \frac{\alpha_{n}^{2} J_{0}^{2}\left(\alpha_{n} \kappa\right)}{J_{0}^{2}\left(\alpha_{n} \kappa\right)-J_{0}^{2}\left(\alpha_{n}\right)}\right]\left[\frac{\phi^{2} \mathrm{X}}{\phi^{2}+\alpha_{n}}\right]\left[\frac{\cosh \left(\lambda_{n} \xi\right)}{\cosh \lambda_{n}}\right] R_{0}\left(\alpha_{n}, \rho\right)$

where $\alpha_{n}$ are roots of

$J_{0}\left(\alpha_{n}\right) Y_{0}\left(\kappa \alpha_{n}\right)-J_{0}\left(\kappa \alpha_{n}\right) Y_{0}\left(\alpha_{n}\right)=0$

In this study, $Y_{0}$ is the Bessel function of the second kind, and

$\lambda_{\mathrm{n}}^{2}=\gamma^{2}\left(\phi^{2}+\alpha_{\mathrm{n}}^{2}\right)$

$R_{0}\left(\alpha_{n} \rho, \alpha_{n}\right)=J_{0}\left(\alpha_{n} \rho\right) Y_{0}\left(\alpha_{n}\right)-J_{0}\left(\alpha_{n}\right) Y_{0}\left(\alpha_{n} \rho\right)$

$\mathrm{X}=\int_{\kappa}^{1} R_{0}\left(\alpha_{\mathrm{n}}, \mathrm{x}\right) d x=\alpha_{n}^{-1}\left[\mathrm{Y}_{0}\left(\alpha_{n}\right)\left\{J_{1}\left(\alpha_{n}\right)-\kappa J_{1}\left(\kappa \alpha_{\mathrm{n}}\right)\right\}\right.$

$\left.-J_{0}\left(\alpha_{\mathrm{n}}\right)\left\{Y_{1}\left(\alpha_{n}\right)-\kappa Y_{1}\left(\kappa \alpha_{\mathrm{n}}\right)\right\}\right]$

The substitution of Eqs. (5a) and (9) in Eq. (6) yields the complete solution shown as follows:

$v(\rho, \xi)=\frac{P_{0}(\phi, \phi \rho)-P_{0}(\kappa \phi, \phi \rho)}{P_{0}(\phi, \kappa \phi)}+$

$+\sum_{n=1}^{\infty}\left[\frac{\pi^{2}}{2} \frac{\alpha_{n}^{2} J_{0}^{2}\left(\alpha_{n} \kappa\right)}{J_{0}^{2}\left(\alpha_{n} \kappa\right)-J_{0}^{2}\left(\alpha_{n}\right)}\right]\left[\frac{\phi^{2} X}{\phi^{2}+\alpha_{n}}\right]\left[\frac{\cosh \left(\lambda_{n} \xi\right)}{\cosh \lambda_{n}}\right] R_{0}\left(\alpha_{n} \rho, \alpha_{n}\right)$

To evaluate the effective utilization of the catalytic pellet, the catalyst effectiveness factor is invariably used. In the present case, the factor can be written as

$\eta=\left(\frac{2}{1-\kappa^{2}}\right) \int_{0}^{1} \int_{\kappa}^{1} v(\rho, \xi) \rho d \rho d \xi$

For convenience, the value can be expressed as the sum of two contributions shown as follows:

$\eta_{1 \infty}=\left(\frac{2}{1-\kappa^{2}}\right)\left[\frac{\left\{\mathrm{Q}_{0}(\kappa \phi, \phi)-\mathrm{Q}_{0}(\phi, \phi)\right\}+\kappa\left\{\mathrm{Q}_{0}(\phi, \kappa \phi)-\mathrm{Q}_{0}(\kappa \phi, \kappa \phi)\right\}}{\mathrm{P}_{0}(\phi, \kappa \phi)}\right]$

$\eta_{1}=\eta_{1 \infty}+\left(\frac{2}{1-\kappa^{2}}\right)\left(\frac{\pi^{2} \phi^{2} \gamma^{2}}{2}\right) \sum_{n=1}^{\infty}\left[1-\left\{\frac{J_{0}\left(\alpha_{n}\right)}{J_{0}\left(\kappa \alpha_{n}\right)}\right\}^{2}\right]^{-1}\left[\frac{\tanh \left(\lambda_{\mathrm{n}}\right)}{\lambda_{\mathrm{n}}^{3}}\right] \alpha_{\mathrm{n}}^{2} \mathrm{X}^{2}$

where

$Q_{0}(a, b)=I_{0}(a) K_{1}(b)-K_{0}(b) I_{1}(a)$

In this study, $\eta_{1 \infty}$ is the effectiveness factor for an infinite hollow cylinder.

\section{Approach 2}

The governing differential equation for an infinite disk is given by

$$
\frac{\partial^{2} v_{2}}{\partial \xi^{2}}-(\gamma \phi)^{2} v_{2}=0
$$

At $\xi=0 ; \frac{\partial v_{2}}{\partial \xi}=0$ for all $\varrho$

At $\xi=1 ; \quad v_{2}=1$ for all $\varrho$

The solution of Eqs. (18) and (19) is given by

$v_{2}(\xi)=\frac{\cosh (\phi \gamma \xi)}{\cosh (\phi \gamma)}$

Introducing the following substitution

$v(\rho, \xi)=\Psi_{2}(\rho, \xi)+v_{2}(\xi)$

to Eqs. (1) and (2) yields

$\left[\frac{\partial^{2} \Psi_{2}}{\partial \rho^{2}}+\frac{1}{\rho} \frac{\partial \Psi_{2}}{\partial \rho}\right]+\frac{1}{\gamma^{2}}\left[\frac{\partial^{2} \Psi_{2}}{\partial \xi^{2}}\right]-\phi^{2} \Psi_{2}=0$

At $\rho=\kappa ; \quad \Psi_{2}=\left(1-v_{2}\right)$

At $\rho=1 ; \quad \Psi_{2}=\left(1-v_{2}\right)$

At $\xi=0 ; \quad \frac{d \Psi_{2}}{d \xi}=0$

At $\xi=1 ; \quad \Psi_{2}=0$

The abovementioned problem is divided into two parts in such a way that only one non-homogeneous boundary condition is retained by the each individual part. As a result,

$\left[\frac{\partial^{2} \Theta_{2 A}}{\partial \rho^{2}}+\frac{1}{\rho} \frac{\partial \Theta_{2 A}}{\partial \rho}\right]+\frac{1}{\gamma^{2}}\left[\frac{\partial^{2} \Theta_{2 A}}{\partial \xi^{2}}\right]-\phi^{2} \Theta_{2 A}=0$

At $\rho=\kappa ; \quad \Theta_{2 A}=\left(1-v_{2}\right)$

At $\rho=1 ; \quad \Theta_{2 A}=0$

At $\xi=0 ; \quad \frac{d \Theta_{2 A}}{d \xi}=0$

At $\xi=1 ; \quad \Theta_{2 A}=0$

and

$\left[\frac{\partial^{2} \Theta_{2 B}}{\partial \rho^{2}}+\frac{1}{\rho} \frac{\partial \Theta_{2 B}}{\partial \rho}\right]+\frac{1}{\gamma^{2}}\left[\frac{\partial^{2} \Theta_{2 B}}{\partial \xi^{2}}\right]-\phi^{2} \Theta_{2 B}=0$

At $\rho=\kappa ; \quad \Theta_{2 B}=0$

At $\rho=1 ; \quad \Theta_{2 B}=\left(1-v_{2}\right)$

At $\xi=0 ; \quad \frac{d \Theta_{2 B}}{d \xi}=0$

At $\xi=1 ; \quad \Theta_{2 B}=0$

Using the separation of variable method for both parts, the equation can written as

$\Theta_{2 \mathrm{~A}}=2 \phi^{2} \sum_{\mathrm{n}=1}^{\infty}\left(\frac{\sin \beta_{n}}{\beta_{n}}\right)\left(\frac{\cos \left(\beta_{n} \xi\right)}{\mu_{n}^{2}}\right)\left[\frac{P_{0}\left(\mu_{n}, \mu_{n} \rho\right)}{P_{0}\left(\mu_{n}, \kappa \mu_{n}\right)}\right]$

$\Theta_{2 \mathrm{~B}}=2 \phi^{2} \sum_{\mathrm{n}=1}^{\infty}\left(\frac{\sin \beta_{n}}{\beta_{n}}\right)\left(\frac{\cos \left(\beta_{n} \xi\right)}{\mu_{n}^{2}}\right)\left[\frac{P_{0}\left(\mu_{n}, \mu_{n} \rho\right)}{P_{0}\left(\mu_{n}, \kappa \mu_{n}\right)}\right]$ 
where

$\mu_{\mathrm{n}}^{2}=\phi^{2}+\beta_{\mathrm{n}}^{2} / \gamma^{2}$

and $\beta_{n}$ are the roots of $\cos \left(\beta_{n}\right)=0$, such that

$\beta_{n}=(2 n-1) \frac{\pi}{2}$

Therefore, the solution of Eq. (22) and associated boundary conditions is given as

$\psi_{2}=2 \phi^{2} \sum_{n=1}^{\infty}\left(\frac{\sin \beta_{n}}{\beta_{n}}\right)\left(\frac{\cos \left(\beta_{n} \xi\right)}{\mu_{n}^{2}}\right)\left[\frac{P_{0}\left(\mu_{n} \rho, \kappa \mu_{n}\right)+\mathrm{P}_{0}\left(\mu_{n}, \mu_{n} \rho\right)}{\mathrm{P}_{0}\left(\mu_{n}, \kappa \mu_{n}\right)}\right]$

The effectiveness factor for an infinite disk is given as $\eta_{2 \infty}=\frac{\tanh (\gamma \phi)}{(\gamma \phi)}$

Therefore, the effectiveness factor using this approach can be written as

$\eta_{2}=\eta_{2 \infty}+\left(\frac{4 \phi^{2}}{1-\kappa^{2}}\right)$

$\sum_{n=1}^{\infty}\left[\frac{\left\{Q_{0}\left(\kappa \mu_{n}, \mu_{n}\right)-Q_{0}\left(\mu_{n}, \mu_{n}\right)\right\}+\kappa\left\{Q_{0}\left(\mu_{n}, \kappa \mu_{n}\right)-Q_{0}\left(\kappa \mu_{n}, \kappa \mu_{n}\right)\right\}}{\beta_{n}^{2} \mu_{n}^{3} P_{0}\left(\mu_{n}, \kappa \mu_{n}\right)}\right]$

\section{RESULTS AND DISCUSSION}

\section{Convergence efficiency}

As discussed previously, different solution methodologies have been suggested for such problems in literature. Making discrete Fourier sine expansion of $\mathrm{v}(\xi)$, Wijngaarden et al. ${ }^{20}$ first derived the expression for the concentration distribution, which was integrated to obtain the following expression for the effectiveness factor,

$\eta=1-\left(\frac{2 \phi^{2}}{1-\kappa^{2}}\right) \sum_{\mathrm{n}=1}^{\infty}\left[\frac{1-\kappa^{2}-2 \chi\left(\kappa \mu_{n}, \mu_{n}\right)}{\beta_{n}^{2} \mu_{n}^{2}}\right]$

where

$\chi(a, b)=\frac{2-\psi(a, b)}{b^{2} P_{0}(a, b)}$

$\psi(a, b)=b Q_{0}(a, b)+a Q_{0}(b, a)$

On the other hand, Gunn ${ }^{21}$ used the standard decomposition approach for deriving the effectiveness factor expression, which shows slow convergence behavior, because the mathematical technique used is probably not suitable to address the present non-homogeneous problem. His expression of the effectiveness factor can be simplified as follows:

$\eta=\left(\frac{4}{1-\kappa^{2}}\right)\left[\sum_{n=1}^{\infty} \frac{J_{0}\left(\kappa \alpha_{n}\right)-J_{0}\left(\alpha_{n}\right)}{J_{0}\left(\kappa \alpha_{n}\right)+\mathrm{J}_{0}\left(\alpha_{n}\right)} \frac{\tanh \left(\lambda_{n}\right)}{\alpha_{n}^{2} \lambda_{n}}+\right.$

$\left.+\sum_{\mathrm{n}=1}^{\infty} \frac{2-\psi\left(\mu_{n}, \kappa \mu_{n}\right)}{\beta_{n}^{2} \mu_{n} P_{0}\left(\mu_{n}, \kappa \mu_{n}\right)}\right]$

To examine the convergence efficiency of different analytical expressions for the effectiveness factor, its value, for a given value of the Thiele modulus, is computed by taking different numbers of terms in the series expansion. The efficient expression for the effectiveness factor will require less number terms and thus will ensure fast convergence. The truncation of the series introduces an error in the computed value of the effectiveness factor. In the present case, the percentage error is defined as follows:

$\%$ Error $=\left(\frac{\eta_{\text {actual }}-\eta_{\text {computed }}}{\eta_{\text {actual }}}\right) \times 100$

The results are presented in Figures 2 to 4 . $x$ is kept at 0.5 , which means that the inner radius is half of the outer one, whereas three different values for $\gamma(=0.1$, 1 , and 10) are chosen. The value of the Thiele modulus $\Phi$ is 10 in these figures. The Thiele modulus is defined using the characteristic length $V_{p} / S_{p}$ shown as follows:

$\Phi=\left[\frac{3(1-\kappa) \gamma_{\mathrm{o}}}{1-\kappa+2 \gamma_{\mathrm{o}}}\right] \phi$

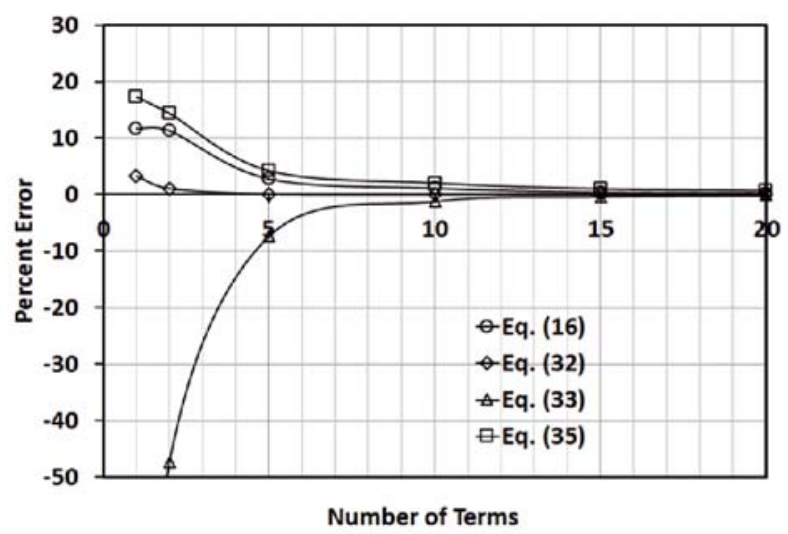

Figure 2. Convergence efficiency of effectiveness factor expressions for $\gamma=0.1, x=0.5, \Phi=10, \eta=0.0972$

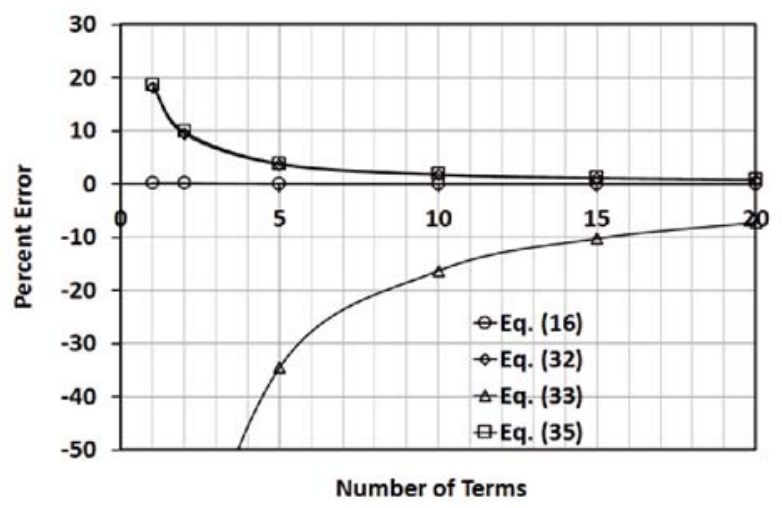

Figure 3. Convergence efficiency of effectiveness factor expressions for $\gamma=1, x=0.5, \Phi=10, \eta=0.0979$

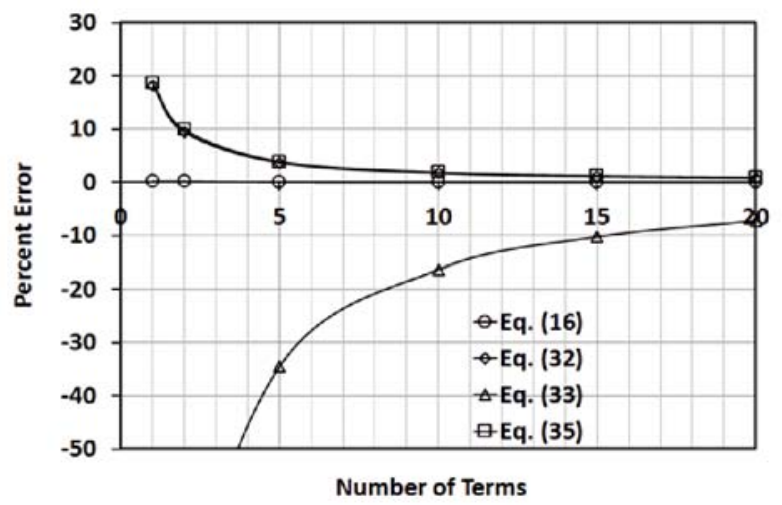

Figure 4. Convergence efficiency of effectiveness factor expressions for $\gamma=10, x=0.5, \Phi=10, \eta=0.0997$ 
The abscissa here is the number of terms used in the summation of the infinite series. In cases where roots of the cosine as well as the Bessel function are required, an equal number of both roots are considered for ease of presentation. On the one hand, for a small value of parameter $\gamma(=0.1)$ in Figure 2, when the cylinder will resemble a disk, the fastest convergence is observed for Approach 2 (Eq. (32)). Even with two terms of the series, a solution of reasonable accuracy is obtained. This result is expected because Eq. (32) uses one-dimensional disk solution as the particular solution, which is in itself a good approximation of the problem at hand. On the other hand, Figure 3 presents the case for $\gamma=1$. The convergence behavior of Eq. (16) is the best, while Eq. (32) shows a better convergence than other expressions reported in literature. The case of a high $\gamma(=10)$ is presented in Figure 4. The geometry resembles a long cylinder. Therefore, the convergence is obviously fastest for the case of Approach 1 (Eq. 16), where the particular solution is based on the solution of an infinite hollow cylinder. In this case, even a single term of the series is sufficient for a solution of reasonable accuracy. The other schemes in the figure are obviously significantly slower. All these results clearly show that the accuracy of the simplified Gunn's expression, represented by Eq. (35), is at best comparable to Eq. (32). By contrast, Eq. (33) shows a poor convergence behavior.

The data of Figure 3 are also presented in Table 1 for clarity. Eq. (16) shows the best convergence behavior, followed by Eq. (32). On the contrary, the error associated with Eq.(35) is high. The poor convergence behavior of Eq. (33) is also clearly evident in this part. Therefore, the particular solution-based approach shows a significantly better convergence behavior than the other approaches proposed in literature. The superiority of the particular solution-based approach has been demonstrated earlier in the context of a solid cylinder as well ${ }^{18}$. With regard to the choice of the two approaches discussed in this paper, the solution strategy itself shows that Approach 1 shows a faster convergence behavior than Approach 2 for finite long hollow cylinders. This finding is due to that the effectiveness factor is computed as a departure from the case of an infinite hollow cylinder. Meanwhile, the use of the analytical expression based on Approach 2 shows a superior convergence behavior for short hollow cylinders, as the effectiveness factor in this case is computed as a departure from the case of an infinite disk.

\section{Effect of shape parameters on effectiveness factor}

At this stage, examining the effect of shape parameters on the effectiveness factor while keeping the amount or the volume of the pellet fixed is important. This statement means that, for a given amount of the catalytic material, the effect of the variation of the catalyst geometry on its utilization is analyzed. Toward this end, the Thiele modulus is defined using the equivalent volume diameter shown as follows

$\phi_{V}=\sqrt{\frac{k_{1}}{4 D_{r}}} D_{V}$

where $D_{V}$ is the equivalent volume diameter defined by

$D_{V}=\left[12\left(1-\kappa^{2}\right) \gamma_{0}\right]^{\frac{1}{3}} R_{0}$

The dependence of the effectiveness factor on the Thiele modulus is shown in Figure 5 a for different values of $\gamma$ and a fixed value of $\varkappa=0.5$, which corresponds to a hollow cylinder with an internal-to-external diameter ratio of 0.5 . The effectiveness factor of the sphere is also presented in the figure for comparison. A hollow cylindrical shape clearly substantially increases the effectiveness factor of the catalyst pellet. The shape parameter $\gamma$ plays an important role in this connection. The general trend shows that a greater departure of $\gamma$ from unity results in a high effectiveness factor. This finding can be clearly observed in Figure 5 b. The ordinate in this figure is defined as

$\%$ Difference $=\frac{100 \times\left(\eta_{\text {hollow cylind. }}-\eta_{\text {sphere }}\right)}{\eta_{\text {sphere }}}$

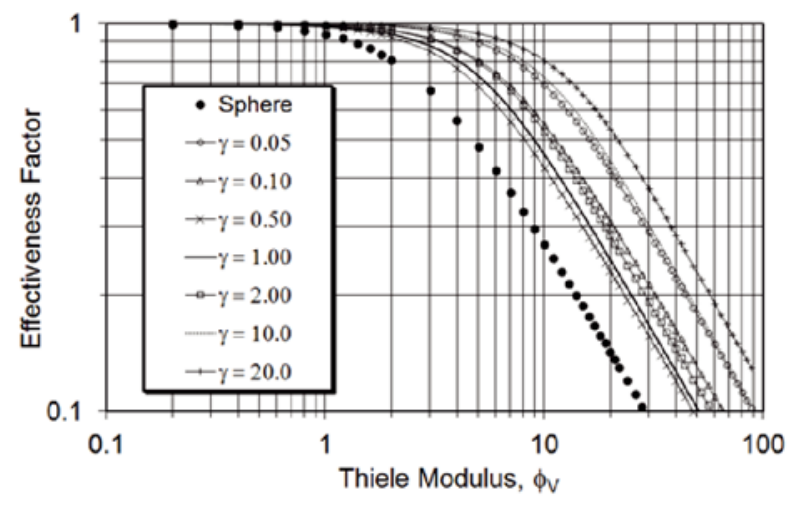

Figure 5a. Dependence of effectiveness factor on the Thiele modulus for $\kappa=0.5$

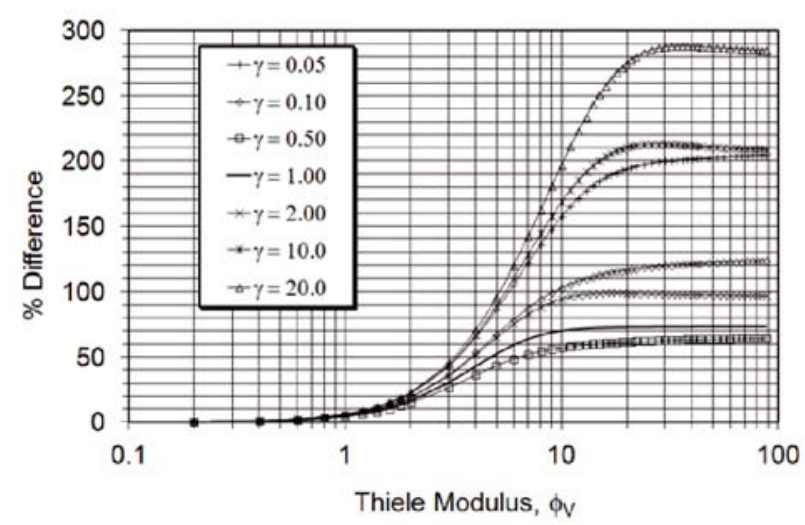

Figure 5b. Percent improvement in effectiveness factor as function of Thiele modulus and $\gamma(\kappa=0.5)$

Table 1. Percentage errors associated with number of terms for different expressions presented in Figure 3

\begin{tabular}{|c|c|c|c|c|}
\hline Terms & Equation (16) & Equation (32) & Equation (33) & Equation (35) \\
\hline 1 & 2.25 & 13.48 & -160.31 & 17.5 \\
\hline 2 & 2.14 & 6.22 & -76.43 & 10.07 \\
\hline 5 & 0.26 & 1.69 & -23.09 & 3.1 \\
\hline 10 & 0.06 & 0.42 & -7.12 & 1.19 \\
\hline 15 & 0.01 & 0.14 & -3.05 & 0.59 \\
\hline 20 & 0 & 0.06 & -1.55 & 0.36 \\
\hline
\end{tabular}


The improvement in the effectiveness factor is as high as $300 \%$ for long hollow cylinders, compared with a solid sphere for the same volume of the catalytic material. A solid cylinder with $\gamma=1$ yields an improvement of nearly $10 \%$ over that of a sphere. However, the same value increases nearly sevenfold for a hollow cylinder. The curves in Figure 5b generally show an increasing trend with the increase in the Thiele modulus. This finding is due to that the internal mass transfer resistance becomes increasingly important at high Thiele modulus. Therefore, the full potential of a hollow catalyst pellet is realized when the diffusional or the internal mass transfer resistance is important.

The dependence of the effectiveness factor on $\gamma$ is shown in Figure 6 for different Thiele moduli and fixed $x$. The figure shows that the effectiveness factor decreases as the Thiele modulus increases. This finding is a clear indication that the internal mass transfer resistance increases with the increase in the Thiele modulus, thereby leading to a decrease in the effectiveness factor. This issue is a main interest in the design of heterogeneous reactors, as a decrease in effectiveness is a measure of a decrease in the effective utilization of the catalyst being used for the conversion. This result consequently leads to a decrease in the conversion level of the reactor. Figure 6 highlights the effect of $\gamma$ on the effectiveness factor. The total volume of the catalyst pellet is held constant for a fixed value of the Thiele modulus in this case. Therefore, the change in the effectiveness factor is solely due to the variation in $\gamma$. Notably, all curves show a minimum, which tends to occur when the $\gamma$ ratio is close to unity. However, a close look on the figure reveals that these curves are asymmetrical around the unity. When $x=0.5$, the effectiveness factor is slightly higher for $\gamma=0.01$ than that for $\gamma=100$. The curve for $x=0.9$ is also shown for the Thiele modulus of 10 . The effectiveness factor is high for high $x$ under the same Thiele modulus. However, a shift occurs in the minimum toward a low $\gamma$. A low value of $\gamma$ represents a hollow ring, whereas a large value of the same represents a long hollow cylinder. From a design standpoint, such a geometrical configuration of the catalyst shape is therefore recommended for achieving a high degree of conversion.

The effect of the shape parameter $x$ is highlighted in Figure 7, which depicts the dependence of the effectiveness factor on $\gamma$ for different values of $x$ when $\phi_{\mathrm{v}}=20$.

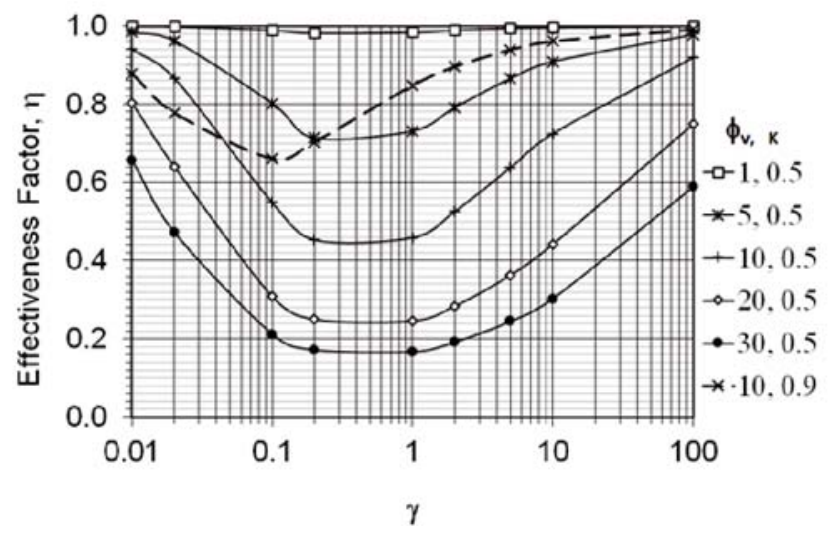

Figure 6. Dependence of the effectiveness factor on $\gamma$ for different values of the Thiele modulus $\left(\phi_{v}\right)$

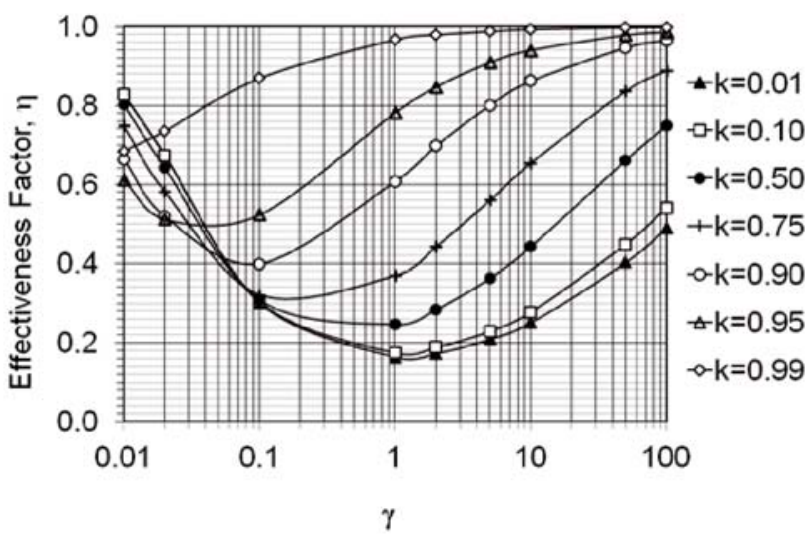

Figure 7. Dependence of the effectiveness factor on the $\gamma$ for different values of $x=0.5$ and a fixed Thiele modulus $\left(\phi_{v}=20\right)$

All curves show a minimum, which tends to progressively shift toward a low $\gamma$ as the value of $x$ decreases. $x$ is in fact a measure of the thickness of the hollow cylinder. A high $x$ means a thin hollow cylinder. A small $x$ represents a hollow cylinder whose inner diameter is very small compared with the outer one, which will be effectively similar to a solid cylinder. This finding means that the minimum value of the effectiveness factor will occur for a solid cylinder. As $x$ increases, the effectiveness factor always shows an increase for all values of $\gamma$. However, the difference is pronounced for long hollow cylinders. For short hollow cylinders, the change in $x$ does not make a significant difference unless they are very thin.

\section{CONCLUSION}

The present strategy of using a particular solution to eliminate the non-homogeneity in the boundary conditions clearly yields efficient expressions for the effectiveness factor for a finite hollow cylindrical catalyst pellet undergoing first-order irreversible reaction. This finding is due to that the case of finite hollow cylindrical geometry is treated as a departure from its corresponding infinite case, which is either an infinite hollow cylinder or a disk. This result explains the improved convergence behavior of Eq. (16) for long hollow cylinders and Eq. (32) for rings. The comparison also shows that, unless $\gamma$ is significantly less than unity, Eq. (16) shows a better convergence behavior than Eq. (32).

The effect of shape parameters, namely, $\gamma$ and $x$, on the effectiveness factor is significant on the effectiveness factor of the pellet. A choice of these parameters can result in several-fold increases in the effectiveness factor as compared with those obtained with a spherical shape or a solid cylindrical shape for a fixed volume of the catalytic material. On the one hand, as far as the effect of $\gamma$ on the effectiveness factor is concerned, a minimum is observed to be close to unity for low values of $x$. Therefore, long cylinders as well as short rings are equally effective for an improved utilization of the catalyst mass. On the other hand, a large $x$ is highly effective in enhancing the effectiveness factor. Choosing a large $x$ however presents practical problems due to the thinness of the catalyst pellet, which will be more prone to breakage when used as packing during reactor operation. 


\section{ACKNOWLEDGMENT}

Authors appreciate the support of the Deanship of Scientific Research at the King Saud University for the Research Group, RGP-1437-003.

\section{NOMENCLATURE}

C - Concentration of reactant in the catalyst pellet

$\mathrm{C}_{0} \quad$ - Concentration of reactant at the surface of the catalyst pellet

$\mathrm{D}_{\mathrm{r}} \quad$ - Reactant diffusivity in the radial direction

$D_{v} \quad$ - Volume equivalent diameter of a non-spherical particle defined by Eq. (39)

$\mathrm{D}_{z} \quad$ - Reactant diffusivity in the axial direction

$\mathrm{H} \quad-$ Height of the catalyst pellet

$\mathrm{k}_{1} \quad$ - First order rate of reaction

$\mathrm{P}_{0}(\mathrm{a}, \mathrm{b})-\mathrm{P}_{0}(\mathrm{a}, \mathrm{b})=\mathrm{I}_{0}(\mathrm{a}) \mathrm{K}_{0}(\mathrm{~b})-\mathrm{I}_{0}(\mathrm{~b}) \mathrm{K}_{0}(\mathrm{a})$ defined by Eq. (5b)

$\mathrm{v}_{1} \quad$ - Particular solution defined by Eq. (5a)

$\mathrm{v}_{2} \quad$ - Particular solution defined by Eq. (20)

$\mathrm{Q}_{0}(\mathrm{a}, \mathrm{b})-\mathrm{Q}_{0}(\mathrm{a}, \mathrm{b})=\mathrm{I}_{0}(\mathrm{a}) \mathrm{K}_{1}(\mathrm{~b})-\mathrm{K}_{0}(\mathrm{~b}) \mathrm{I}_{1}(\mathrm{a})$ defined by Eq. (17)

r $\quad$ - Radial coordinate

$\mathrm{R}_{\mathrm{i}} \quad$ - Inner radius

$\mathrm{R}_{0}(\mathrm{a}, \mathrm{b})-\mathrm{R}_{0}(\mathrm{a}, \mathrm{b})=\mathrm{J}_{0}(\mathrm{a}) \mathrm{Y}_{0}(\mathrm{~b})-\mathrm{J}_{0}(\mathrm{~b}) \mathrm{Y}_{0}(\mathrm{a})$ defined by eq. (11b)

$\mathrm{R}_{\mathrm{o}} \quad$ - Outer radius

$\mathrm{S}_{\mathrm{p}} \quad$ - Surface area of the catalyst particle

$\mathrm{v}$ - Dimensionless reactant concentration in the pellet defined by Eq. (3)

$\mathrm{V}_{\mathrm{p}} \quad$ - Volume of the catalyst particle

z $\quad$ - Axial coordinate

\section{Greek Symbols}

$\alpha \quad-$ Roots of $\mathrm{J}_{0}\left(\alpha_{\mathrm{n}}\right) \mathrm{Y}_{0}\left(\kappa \alpha_{\mathrm{n}}\right)-\mathrm{J}_{0}\left(\kappa \alpha_{\mathrm{n}}\right) \mathrm{Y}_{0}\left(\alpha_{\mathrm{n}}\right)=0$

$\beta \quad-$ Roots of $\cos \left(\beta_{n}\right)=0$ given as $(2 n-1) \frac{\pi}{2}$

$x \quad-$ Ratio of inner to outer radii as defined in Eq. (3)

$\phi \quad-$ Thiele modulus defined in Eq. (3)

$\phi_{V} \quad$ - Thiele modulus defined in Eq. (38)

$\Phi \quad$ - Thiele modulus defined using $(\mathrm{Vp} / \mathrm{Sp})$ as the characteristic dimension in Eq. (37)

$\gamma \quad-$ Ratio defined in Eq. (3) that equals ratio $\left(\mathrm{H} / 2 \mathrm{R}_{\mathrm{o}}\right)$ for isotropic catalyst pellet

$\lambda \quad-$ Parameter defined by Eq. (11a)

$\eta \quad$ - Effectiveness factor

$\mu \quad$ - Parameter defined in Eq. (29a)

$\rho \quad$ Dimensionless radial coordinate defined

by Eq. (3)

$\xi \quad$ Dimensionless axial coordinate defined by Eq. (3)

$\psi(a, b)-\psi(a, b)=b_{0}(a, b)+a Q_{0}(b, a)$ defined by Eq. (34b)

$\chi(\mathrm{a}, \mathrm{b})-\chi(\mathrm{a}, \mathrm{b})=\frac{2-\psi(\mathrm{a}, \mathrm{b})}{\mathrm{b}^{2} \mathrm{P}_{0}(\mathrm{a}, \mathrm{b})}$ defined by Eq. (34a)

\section{Subscripts}

1 - Refers to Method/Approach 1

2 - Refers to Method/Approach 2

M - Refers to the number of root in the series summation

\section{LITERATURE CITED}

1. Morbidelli, M., Servida, A. \& Varma, A. (1982). Optimal catalyst activity profiles in pellets. 1 . The case of negligible external mass transfer resistance. Ind. Eng. Chem. Fundam. 21(3), 278-284. DOI: 10.1021/i100007a015.

2. Wu, H., Brunovska, A., Morbidelli, M. \& Varma, A. (1990). Optimal catalyst acitivity profiles in pellets-VIII. General nonisothermal reacting systems with arbitrary kinetics. Chem. Eng. Sci. 45(7), 1855-1862. DOI: 10.1016/0009-2509(90)87061-V.

3. Wu, H., Yuan, Q. \& Zhu, B. (1990). An experimental study of optimal active catalyst distribution in pellets for maximum selectivity. Ind. Eng. Chem. Res. 29(9), 1771-1776. DOI: 10.1021/ie00105a006.

4. Baratti, R., Gavriilidis, A., Morbidelli, M. \& Varma, A. (1994). Optimization of a nonisothermal nonadiabatic fixed-bed reactor using dirac-type silver catalysts for ethylene epoxidation. Chem. Eng. Sci. 49(12), 1925-1936. DOI: 10.1016/00092509(94)80077-4.

5. Morbidelli, M., Gavriilidis, A. \& Varma, A. (2001). Catalyst design, optimal distribution of catalyst in pellets, reactors, and membranes (1st ed.). Cambridge, U.K.: Cambridge University Press.

6. Hwang, S., Linke, P. \& Smith, R. (2004). Heterogeneous catalytic reactor design with optimum temperature profile II: application of non-uniform catalyst. Chem. Eng. Sci. 59(20), 4245-4260. DOI: 10.1016/j.ces.2004.05.036.

7. Wei, J. (2011). Catalyst designs to enhance diffusivity and performance-I: Concepts and analysis. Chem. Eng. Sci. 66 (19), 4382-4388. DOI: 10.1016/j.ces.2011.02.010.

8. Wang, G. \& Coppens, M.-O. (2010). Rational design of hierarchically structured porous catalysts for autothermal reforming of methane. Chem. Eng. Sci. 65(7), 2344-2351. DOI: 10.1016/j.ces.2009.09.079.

9. Wang, G., Johannessen, E., Kleijn, C.R., de Leeuw, S.W. \& Coppens, M.O. (2007). Optimizing transport in nanostructured catalysts: A computational study. Chem. Eng. Sci. 62(18-20), 5110-5116. DOI: 10.1016/j.ces.2007.01.046.

10. Ying, J.Y. \& Martinez, J.G. Mesostructured Zeolitic Materials and Methods of Making and Using the Same, Google Patents, 2009.

11. Asif, M. (2013). Conversion Enhancement of Fixed-Bed Reactors Using Two-Dimensional Hollow Cylindrical Catalyst Pellet. Int. J. Chem. React. Eng. 11(1), 159. DOI: 10.1515/ijcre-2012-0038.

12. Fogler, H.S. (2011). Essentials of Chemical Reaction Engineering (1st ed.). Upper Saddle River, New Jersey: Prentice Hall.

13. http://pxhl.cn/en/pro_bigpic.asp?id=23

14. http://www.dupont.com/products-and-services/consulting-services-process-technologies/brands/sustainable-solutions/sub-brands/ clean-technologies/uses-and-applications/mecs-catalyst.html

15. http://www.topsoe.com/products/catalysts

16. http://www.matrostech.com/ccatalysts.html]

Asif, M. (2015). Retrofitting of Fixed-Bed Heterogeneous Reactors for Glucose Isomerization. Chem. Eng. Commun. 202 (11), 1547-1556. DOI: 10.1080/00986445.2014.959587.

17. Asif, M. (2015). Retrofitting of Fixed-Bed Heterogeneous Reactors for Glucose Isomerization. Chem. Eng. Commun. 202 (11), 1547-1556. DOI: 10.1080/00986445.2014.959587.

18. Asif, M. (2004). Efficient Expressions for Effectiveness Factor for a Finite Cylinder. Chem. Eng. Res. Des. 82 (5), 605-610. DOI: http://dx.doi.org/10.1205/026387604323142658

19. Ozisik, M.N. (1980). Heat Conduction (2nd ed.). New York: John Wiley.

20. Wijngaarden, R.J., Kronberg, A. \& Westerterp, K.R. (1998). Industrial Catalysis: Optimizing Catalysts and Processes (1st ed.Wiley-VCH Verlag GmbH.

21. Gunn, D.J. (1967). Diffusion and chemical reaction in catalysis and absorption. Chem. Eng. Sci. 22 (11), 1439-1455. DOI: http://dx.doi.org/10.1016/0009-2509(67)80071-X 